\title{
Гетерогенная интеграция с помощью групповой сварки кристалл-пластина - эффективный подход к 3D-интеграции микросхем
}

\author{
Д. Суханов ${ }^{1}$, В. Команов
}

УДК 621.382 | ВАК 05.27 .06

\begin{abstract}
До недавнего времени основной целью совершенствования микроэлектронных устройств было наращивание их возможностей в области хранения и обработки данных. Эти возможности достигались прежде всего уменьшением топологических норм и, соответственно, увеличением плотности упаковки элементарных ячеек в монолитном кристалле микросхемы. Теперь же на первый план выходят потребности таких областей применения электроники, как информационно-коммуникационные технологии, дополненная реальность, автономные транспортные средства, среды для обработки больших данных и т. п. Эти приложения требуют создания электронных компонентов нового типа, в которых акцент делается не на емкость памяти и скорость вычислений, а на расширение функционала единичной микросхемы. Такие устройства включают в себя материалы и структуры, которые невозможно или крайне проблематично объединить средствами обычных для сегодняшнего дня технологических процессов и установок. При этом не снимается традиционная задача ускорения циклов разработки и развертывания массового производства, что создает дополнительные проблемы как для проектировщиков микросхем, так и для технологов, и для производителей оборудования.
\end{abstract}

твечая на новые потребности, электронная отрасль продвигается по пути интеграции отдельных технологий в единый производственный процесс. Возникают новые подходы к построению многокристальных микросхем с одновременным изменением конструкции составляющих их кристаллов, а реализация этих подходов становится возможной благодаря появлению все более прецизионного оборудования, росту скорости обработки информации и внедрению в управление процессами элементов искусственного интеллекта. Сегодня можно говорить об очередной революции, произошедшей в полупроводниковой промышленности - о внедрении в практику разработки и производства микроэлектроники принципа гетерогенной интеграции, означающей вытеснение традиционного литографического масштабирования новыми типами и способами межсоединений и передовыми технологиями сборки микроэлектронных устройств.

ООО «Остек-ЭК», главный специалист поддержки группы пресейлинженеров Технического управления, Sukhanov.D@ostec-group.ru. ООО «Остек-ЭК», инженер.
Гетерогенная интеграция как новая ступень развития в конструировании и производстве микроэлектронных устройств опирается на совокупность нескольких технологических новаций, появившихся в последнее десятилетие. Речь идет, в частности, о технологиях3D-интеграции, появившихся сравнительно недавно, но уже широко применяемых в крупносерийном производстве. Также кэтапу массового применения переходят некоторые передовые технологии корпусирования, такие как корпусирование с разветвлением на уровне пластины (Fan-out wafer-level packaging, FOWLP) и интерпозеры. В зависимости от архитектуры устройства и желаемого уровня интеграции, ведущую роль будут играть несколько методов интеграции, реализуемых на разных производственных уровнях. В частности, повышение требований к минимальной задержке и низкому энергопотреблению подтолкнет индустрию к новым концепциям проектирования микросхем, где решающее значение будет иметь объединение в одном кристалле нескольких функций: сенсорики, фотоники, радиочастотной связи и даже устройств питания. Другими словами, ключевой тенденцией в конструировании микросхем будет функциональная интеграция. 


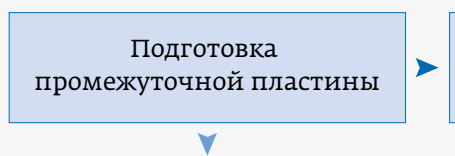

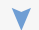

\begin{tabular}{|c|}
\hline Стеклянная \\
или кремниевая \\
пластина \\
$\vee$
\end{tabular}

Нанесение

адгезивного слоя

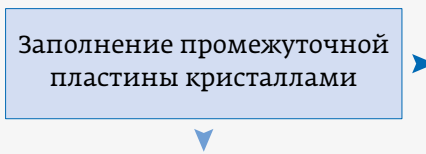

V

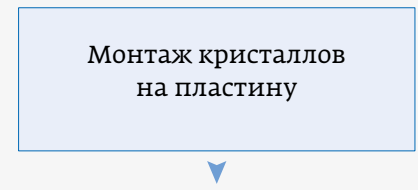

Полностью заполненная пластина

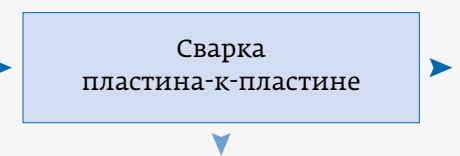

Y

Совмещение рабочей пластины и перевернутой заполненной пластины

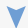

Процесс сварки

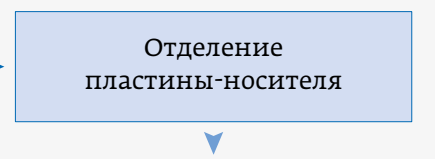

V

Отделение пластины-носителя

\section{V}

Очистка поверхности

\section{Рис. 1. Последовательность операций процесса групповой сварки D2W}

Безусловно, основную роль в новых производствах, как и прежде, будет играть обработка кремния. Однако кремний сам по себе не способен обеспечить всей необходимой функциональности, ради которой появилась и воплощается идея гетерогенной интеграции. Поэтому обязательными и все более важными станут технологии передачи материалов и функциональных микроэлектронных узлов между этапами общего технологического процесса, в котором обработка кремния соединена с нанесением слоев из иных материалов и сборкой структур, решающих вычислительные задачи, с другими структурами, предназначенными для их питания, коммутации и обеспечения первичными сигналами в рамках единой полностью функциональной системы на кристалле (СнК).

Одним из важнейших процессов при объединении составляющих элементов гетерогенно интегрированной конструкции является сварка. Это не новый процесс для микроэлектронной индустрии - изготовители полупроводниковых пластин, а также производители устройств, выполненных по уже освоенным интеграционным технологиям, используют в массовом производстве сварку пластин: прежде всего такие ее разновидности, как сварка сплавлением и гибридная сварка; в последнее время она начинает применяться и в корпусировании.

Однако отработанная технологическая цепочка процесса сварки пластина-к-пластине (W2W, wafer-to-wafer) не может быть прямо применена к задачам, которые должны решаться при помощи гетерогенной интеграции. Она возможна лишь в том случае, когда согласованы размеры кристаллов, сформированных на свариваемых пластинах, или согласован шаг их расположения по обеим осям поверхности пластины. Это требование легко выполнимо для многих классов микроэлектронных устройств, например, для стековых сборок запоминающих устройств или для датчиков изображения. Но гетерогенная система по самой своей сути должна объединять на одной базовой кремниевой подложке разные кремниевые узлы, составные полупроводники для фотонных и силовых устройств или ВЧ-фильтры, поступающие с разных заводов на пластинах различного размера. В этом случае мы имеем дело с интеграцией на уровне микросхемы: на каждый кристалл в неразрезанной еще базовой пластине надо установить один, а часто - несколько кристаллов других размеров, созданных по другой технологии из других материалов. Этим определятся и иной, по сравнению с W2W, тип процесса сварки. Теперь это процесс типа кристалл-к-пластине - по-английски dieto-wafer (D2W).

Такой процесс существует и успешно применяется преимущественно на начальном этапе производства микросхем (front-end), где он организован как последовательный монтаж одиночных кристаллов на пластине с использованием сварки сплавлением или гибридной сварки. Его недостаток в том, что во многих случаях обработка D2W занимает несколько часов производственного процесса, в течение которых поверхность пластины подвержена возможным загрязнениям, что делает затруднительным применение чувствительных к ним технологий, таких как сварка сплавлением. Эту опасность можно предупредить, если все критические операции производятся в единой технологической установке с контролируемой атмосферой, где могут быть выполнены требования по содержанию загрязняющих частиц, соответствующие классу 1 ИСО. Однако проблема окончательно не решается и в этом случае: длительный процесс последовательной установки кристаллов на пластину и затем последовательные перемещения сварочной головки над пластиной неизбежно окажут негативное влияние на выход годных.

Таким образом, обычное оборудование для процесса типа D2W непригодно для реализации гетерогенной интеграции. Так как же решить эту задачу?

\section{ГРУППОВАЯ СВАРКА КРИСТАЛЛ-К-ПЛАСТИНЕ}

Единственное логичное решение - комплексный подход, в рамках которого процесс должен быть разделен 


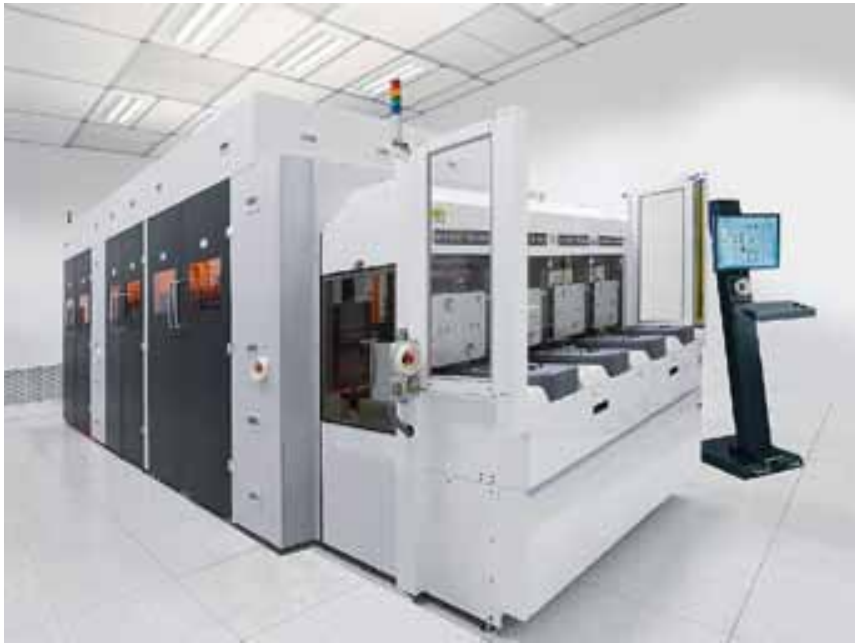

Рис. 2. Автоматизированный производственный комплекс EVC GEMINI ${ }^{\circledR}$. Источник: https://www.evgroup.com/ products/bonding/permanent-bonding-systems/gemini/

на этап размещения кристаллов и этап групповой сварки. Это позволит обеспечить высокую производительность процесса сварки, не увеличивая чрезмерно сложность оборудования. Последовательность операций процесса групповой сварки D2W показана на рис. 1.

Первым этапом является подготовка и защита специальной пленкой поверхности пластины с кристаллами, предназначенными к переносу. Защищают ту поверхность, на которой будет проводиться сварка методом сплавления; назначение защиты-предотвращение засорения пластины при резке, в ходе которой обильно образуются механические частицы, которые могут стать причиной поверхностных дефектов нанометровых структур. Помимо того, что защитная пленка должна

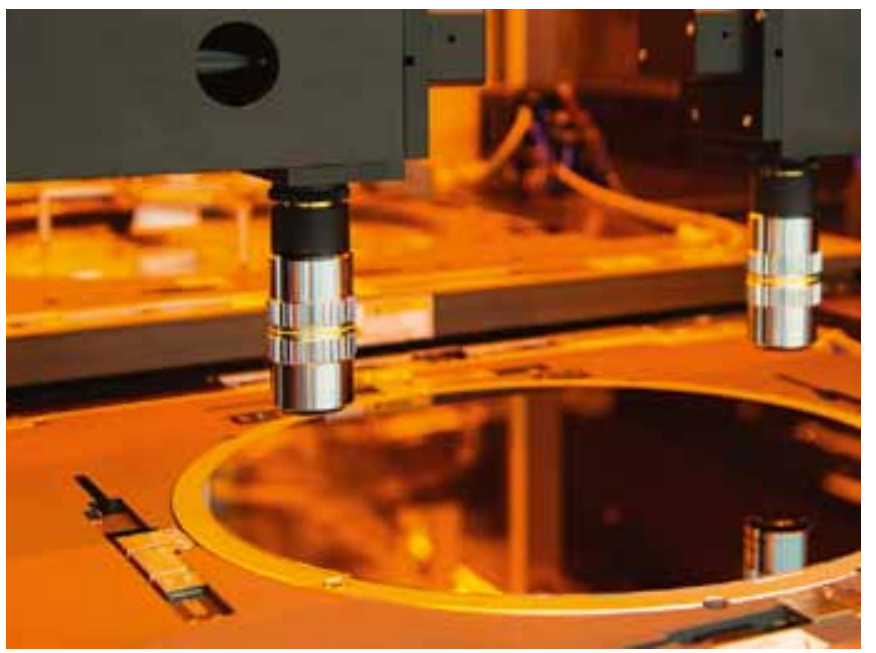

Рис. 3. Система совмещения последнего поколения EVG SmartView ${ }^{\circledR}$ NT3. Источник: www.evgroup.com бытьгладкой и иметь однородную толщину, она должна легко удаляться и не оставлять после себя никаких органических остатков.

После резки каждый кристалл при помощи оборудования, используемого в освоенных сегодня процессах D2W, помещается на оснастку - промежуточный носитель. В зависимости от конструкции собираемого изделия и характера процесса сборки требуемая точность совмещения может варьироваться от долей до нескольких единиц микрометров.

По окончании этих подготовительных операций базовая пластина и промежуточный носитель с закрепленными кристаллами загружаются в полностью автоматизированный производственный комплекс EVG GEMINI (рис. 2). Таким образом, все этапы их совместной обработки проходят в атмосфере с контролируемой концентрацией частиц.

После удаления защитной пленки пластина и кристаллы на промежуточном носителе проходят одинаковую процедуру активации и очистки перед процессами совмещения и сварки, которые, так как теперь кристаллы собраны в единую сборку с нужным шагом, могут проводиться на оборудовании для сварки W2W

Промежуточный носитель переворачивается, обращая кристаллы свариваемой поверхностью к базовой пластине, после чего выполняется процесс совмещения пластин при помощи системы последнего поколения EVG SmartView ${ }^{\circ}$ T3 (рис. 3).

Приведенные в соприкосновение кристаллы на носителе и базовая пластина подвергаются небольшому нагреву и сжатию, чем достигается выравнивание кристаллов по высоте, необходимое для корректного выполнения последующих операций. Проводится сварка, после чего промежуточный носитель отсоединяется от сваренного пакета, и обе пластины очищаются и выгружаются из сварочного кластера, переходя на следующие этапы обработки.

Во врезке представлен обзор параметров и операций, определяющих характеристики группового процесса соединения D2W.

Практическая отработка описанного процесса с использованием различных комбинаций материалов доказала эффективность применения этой технологии для кристаллов различной формы с размерами, лежащими в пределах от 800×800 мкм до 10×10 мм. Кристаллы больших размеров из этого диапазона обычно кремниевые, меньшие размеры кристаллов характерны для составных полупроводников, таких как InP, GaAs, GaN или SiC.

Рассмотрим некоторые существенные особенности отдельных этапов процесса групповой сварки D2W, в основном в той части, где он отличается от уже хорошо известных процессов W2W и последовательного D2W. 


\section{Параметры и операции, определяющие групповой процесс D2W}

Свойства кристалла:

- шероховатость поверхности;

- размер;

- качество кромки;

- неравномерность толщины.

Свойства базовой пластины:

- шероховатость поверхности;

- неравномерность медного слоя (при использовании гибридного процесса сварки);

- размер контактных площадок.

Установка кристаллов:

- точность монтажа (X, Y);

- поворот кристалла;

- температура прихватки.

Процесс сварки:

- активация поверхности пластины и кристаллов;

- совмещение;

- очистка;

- температура, усилие прижима и время.

Свойства промежуточного носителя:

- материал промежуточного носителя;

- неравномерность и толщина слоя адгезива.

Демонтаж и очистка:

- выбор технологии демонтажа;

- очистка растворителем;

- время очистки;

- применимость растворителя

\section{ПОДГОТОВКА ПРОМЕЖУТОЧНОГО НОСИТЕЛЯ}

Основной проблемой подготовки к внедрению групповой сварки D2W является создание эффективного группового носителя кристаллов. Помимо безусловного обеспечения безопасного удержания кристаллов во время очистки и подготовки к последующему этапу сварки, при его проектировании необходимо учитывать несколько других аспектов, значимых для успешного прохождения всего процесса в целом

С самого начала должен быть выбран один из нескольких методов снятия кристаллов с промежуточного носителя, так как в первую очередь им определяется материал, из которого этот носитель должен быть изготовлен. Тип и толщину клеевого материала для монтажа кристаллов выбирают с таким расчетом, чтобы гарантированно обеспечить их прилегание к базовой пластине. Именно этим требованием определяется выбор клея в качестве материала для установки кристаллов на подложке-носителе: деформация эластичного и достаточно толстого слоя этого материала скомпенсирует разницу высот отдельных кристаллов и дефекты плоскостности базовой пластины в момент их соприкосновения. Очень важно также, чтобы клеевой слой был оптимизирован в соответствии с требованиями к точности совмещения, поскольку при групповой сварке может происходить смещение кристаллов, которое необходимо контролировать.

\section{ЗАПОЛНЕНИЕ НОСИТЕЛЯ}

После подготовки промежуточного носителя его заполнение осуществляется с помощью обычного оборудования для монтажа кристаллов D2W, в частности, системы автоматического монтажа компонентов с гранитным основанием Tresky T-8000-G (рис. 4).

Операция выполняется с использованием общего или местного совмещения - в зависимости от требуемой точности позиционирования перемещаемых кристаллов на базовой пластине. Точность общего совмещения, определяемая точностью позиционирования столика установки монтажа кристаллов, обычно бывает достаточной для тех случаев, когда речь идет о монтаже полупроводникового кристалла на структурированную подложку микросхемы, так как формирование рисунка на ней выполняется с помощью литографии на более позднем этапе процесса.

При 3D-интеграции со сборкой стеков с помощью сплавления и гибридного соединения допустимая ошибка совмещения не должна превышать 1 мкм (3б), что во3можно только при использовании промежуточного носителя с литографическими метками, указывающими расположение каждого кристалла, и процесса совмещения при помощи этих меток.

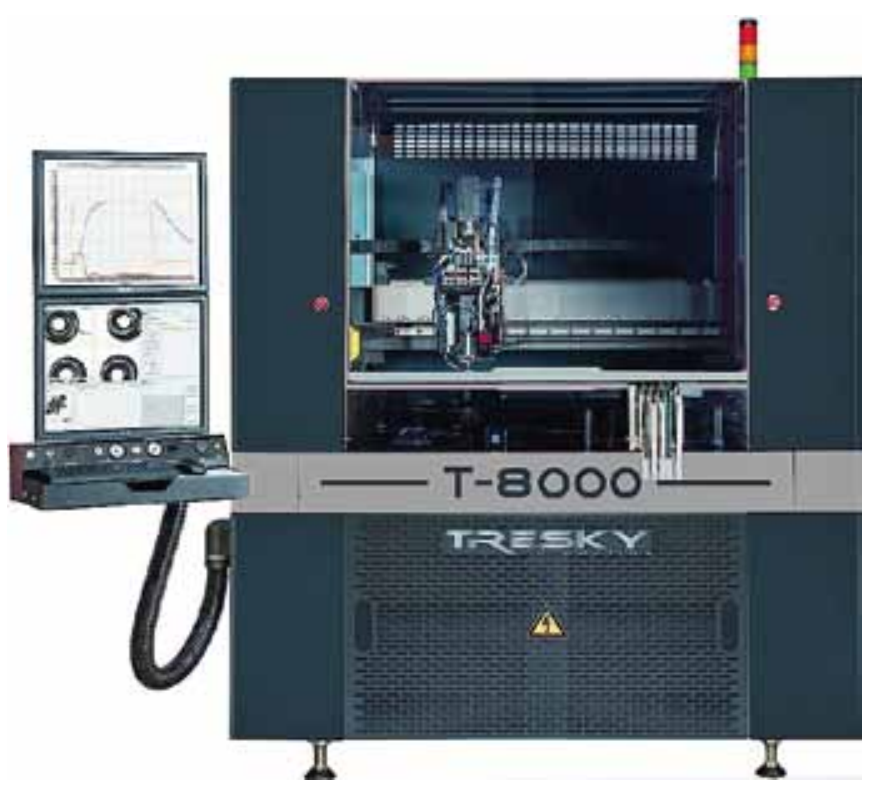

Рис. 4. Система автоматического монтажа компонентов Т-8000-С. Источник: https://www.tresky.de/en/ products/t-8000-g/\#\&gid=lightbox-group-137opid=0 


\section{СВАРКА ПЛАСТИНА-К-ПЛАСТИНЕ}

После заполнения промежуточного носителя сварка кристаллов с базовой пластиной выполняется с использованием стандартного оборудования для сварки W2W. Общий процесс D2W допускает широкий выбор материалов и методов сварки, включая применение любыхадгезивов, низкотемпературных паяных соединений, которые обычно используются для установки выводов из припоя (бампов), а также для термокомпрессионного соединения металлов, например золото-золото или медь-медь, тем самым позволяя избежать недостаточного заполнения поверхности. Для получения высокого выхода годных при термокомпрессионном соединении требуется минимальная шероховатость поверхности металла.

При осуществлении 3D-интеграции методом групповой сварки сегодня используются методы сварки сплавлением или гибридной сварки. В этом случае кристаллы на промежуточном носителе и базовая пластина должны быть очищены и активированы плазмой до процесса сварки.

Плазменная активация необходима для понижения температуры, которая потребуется для соединения кристаллов с базовой пластиной. Процесс низкотемпературной плазменной активации модифицирует поверхность кристаллов и пластины, и в ходе очистки с участием воды на них образуется большое количество гидроксильныхгрупп, являющихся основой для формирования водородных связей между материалами кристаллов и пластины; так достигается их начальное скрепление, что важно для обеспечения высокого уровня выхода годных после сварки. Однако водородные связи сравнительно непрочны, поэтому, получив это предварительное соединение, следует сразу переходить к термической обработке. Фактически, эта обработка является промежуточным этапом процесса сварки: при нагреве до температуры в пределах $250^{\circ} \mathrm{C}$ связь между кристаллами и базовой пластиной заметно упрочняется, но адгезионный слой на промежуточном носителе еще сохраняет свои свойства. После этой стадии проводится отсоединение носителя, после чего следует переход к завершающему этапу - финальной термической обработке, включая приложение механического усилия в процессе гибридной сварки.

\section{ОТДЕЛЕНИЕ ПРОМЕЖУТОЧНОГО НОСИТЕЛЯ}

Для процесса отделения промежуточного носителя доступен обширный спектр методов, использующих световое, силовое либо тепловое воздействие. Применительно кгрупповому процессу D2W надо иметь ввиду, что в момент отделения сварка еще не завершена, сварное соединение не имеет финальной прочности; поэтому демонтаж промежуточного носителя должен производиться мягко: сила, приложенная в вертикальном направлении, должна быть небольшой, чтобы избежать повреждения соединений и отрыва кристаллов от базовой пластины.
Понятно, что прямой механический демонтаж создает значительные риски. В ходе отработки технологии хорошие результаты были получены при термическом демонтаже методом сдвига, а также при использовании для отделения носителя излучения ультрафиолетовых твердотельных лазеров с диодной накачкой.

Для реализации первого из этих процессов роль адгезионного слоя на промежуточном носителе играют термопластичные клеи. Стек нагревают выше температуры размягчения клея, после чего носитель и базовую пластину горизонтально сдвигают относительно друг друга при непрерывном контроле и управлении скоростью и усилием сдвига.

Если для отделения промежуточного носителя планируется использовать лазерное излучение, то носитель должен быть изготовлен из стекла и покрыт специальным адгезивным слоем, который обеспечивает разделение пластин с малым усилием. Гальваносканер осуществляет точный контроль луча, что позволяет лазеру избирательно обрабатывать определенные участки пластины; таким образом можно засвечивать лазером ограниченные области носителя, вплоть до участков под единичными кристаллами. Следует отметить, что перед тем, как поступить на второй этап групповой термической обработки, необходимой для завершения процесса сварки, в обоих вариантах выполнения демонтажа промежуточный носитель и базовая пластина очищаются для удаления любых органических загрязнений.

\section{РЕКОМЕНДАЦИИ ПО СОВМЕЩЕНИЮ}

В процессах сварки пластина-к-пластине уже достигнута точность совмещения менее 100 нм (3б). Точность установки кристаллов на базовую пластину при групповой сварке по описанной здесь технологии в основном определяется двумя операциями (одна из которых отсутствует при сварке пластин, а другая - носит существенно отличный характер): размещением кристаллов на промежуточном носителе и совмещением промежуточного носителя с базовой пластиной по принципу W2W, но при возможном индивидуальном сдвиге кристаллов во время температурного воздействия (отжига). При этом доминирующее значение имеет точность выполнения первой из этих двух операций, влияющая на формирование производственного брака.

Как уже сказано, установка кристаллов на промежуточный носитель выполняется на оборудовании для процессов D2W. Типичным значением точности совмещения для представленного сегодня на рынке оборудования этого класса является значение в 1 мкм. Эту величину следует рассматривать как ограничение, но степень ее значимости не всегда одинакова, она зависит от задач, которые ставятся перед технологией в конкретных случаях ее применения.

Так, если говорить о таких задачах, как корпусирование микросхем памяти или процессоров, или изготовление 
интерпозеров, когда шаг контактов обычно находится в диапазоне от 5 до 10 мкм, то значения допустимой суммарной ошибки совмещения лежат в пределах возможностей технологии групповой сварки D2W. Для других применений, где необходимо совмещение с погрешностью значительно ниже 1 мкм - таких, например, как производство датчиков изображения, - необходимо использовать либо сварку методом сплавления или гибридную сварку, либо установка кристалла должна быть передвинута на более ранние стадии технологического процесса, следовательно, межсоединения будут выполнены после процесса монтажа кристаллов. Более того, субмикронная точность совмещения переходных отверстий может быть получена, если использовать совмещение по индивидуальным литографическим меткам, что позволит повысить точность совмещения на доступных установках монтажа кристаллов. Наконец, для компонентов, которые используются в телекоммуникационных или фотонных устройствах, структурирование подложки может быть произведено после переноса и сварки интегрируемых кристаллов, что даст наилучшее совмещение межсоединений с их контактными площадками.

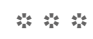

Таким образом, в полупроводниковой промышленности продолжается переход кгетерогенной интеграции для увеличения производительности и расширения возможностей устройств. Это повышает уровень сложности конструкций и производственных процессов, требуя дальнейшего развития технологий трансфера материалов и функциональных кристаллов.

Процесс групповой сварки D2W - это идеальный подход, обеспечивающий высокую производительность при сохранении сложности сварочного оборудования для пластин на приемлемом уровне. Активация поверхностей с помощью плазменной обработки, процессы переноса и сварки кристаллов с пластиной, а также отделение кристаллов от промежуточного носителя и очистка полученной 3D-интегрированной структуры - все это реализовано в автоматической кластерной системе, гарантирующей прохождение процесса в атмосфере с контролируемым содержанием загрязняющих частиц и за счет этого позволяющей достичь высокого значения выхода годных изделий.

Коллективный процесс сварки D2W может быть реализован для различных размеров кристаллов и материалов, таких как кремний или составные полупроводники, в нем могут быть использованы различные методы сварки, что делает этот процесс универсально подходящим для известных и перспективных задач гетерогенной интеграции.

В статье использован материал "Heterogeneous integration by collective die-to-wafer bonding" by Thomas Uhrmann, Jürgen Burggraf, Mariana Pires, Martin Eibelhuber [EV Group]. - http://fbs.advantageinc.com/chipscale/ nov-dec_2018/index.htm|\#12

\section{КНИГИ ИЗДАТЕЛЬСТВА «ТЕХНОСФЕРА»}

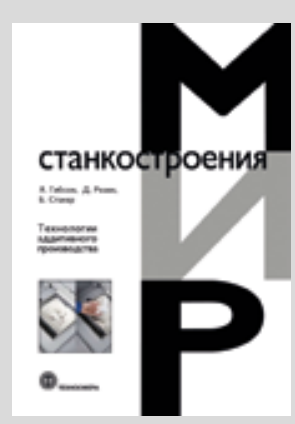

Цена 2600 руб.

\section{ТЕХНОЛОГИИ АДДИТИВНОГО ПРОИЗВОДСТВА ТРЕХМЕРНАЯ ПЕЧАТЬ, БЫСТРОЕ ПРОТОТИПИРОВАНИЕ И ПРЯМОЕ ЦИФРОВОЕ ПРОИЗВОДСТВО}

\author{
Гибсон Я., Розен Д., Стакер Б.
}

При поддержке Департамента станкостроения и инвестиционного машиностроения

Muнnромторга Россuи

Перевод с англ. под ред. д.ф.-м.н. профессора Шишковского И.В.

Книга посвящена новейшим технологиям, которые дают возможность на основе данных о виртуальных моделях твердых тел изготавливать физические модели в результате быстрых и легких производственных процессов.

Авторы книги - признанные специалисты в области аддитивных технологий, имеющие многолетний опыт работы и исследований. Первое издание задумывалось как базовый учебник, объединивший все литературные источники, посвященные целям и задачам аддитивного производства (АП). Второе издание существенно переработано и дополнено, новая информация включена в дополнительные разделы и главы.

Разработчики АП и представители промышленности найдут полезные сведения в этой книге, поскольку она поможет понять состояние дел в отрасли и укажет возможности для дальнейших исследований. Издание предназначено также для преподавателей, студентов и аспирантов, изучающих аддитивное производство, может быть использовано в качестве автономного курса или как модуль в большой программе по технологии производства.

\section{КАК ЗАКАЗАТЬ НАШИ КНИГИ?}

$\bowtie$ 125319, Москва, а/я 91; \(495) 234-0110; 骂 (495) 956-3346; knigi@technosphera.ru, sales@technosphera.ru 